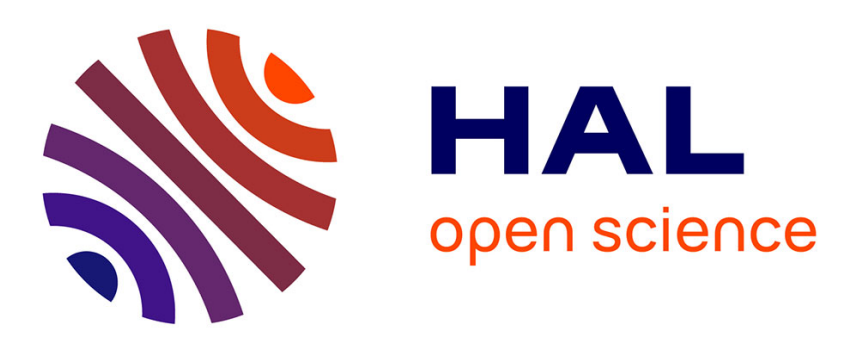

\title{
Relative contributions to the emission within bundles of carbon nanocone emitters
}

\author{
Darius Mofakhami, Philippe Dessante, Philippe Teste, Romaric Landfried, \\ Tiberiu Minea, Benjamin Seznec, J.-P Mazellier, P.-L Gautherin
}

\section{- To cite this version:}

Darius Mofakhami, Philippe Dessante, Philippe Teste, Romaric Landfried, Tiberiu Minea, et al.. Relative contributions to the emission within bundles of carbon nanocone emitters. 33th International Vacuum Nanoelectronics Conference (IVNC 2020), Jul 2020, Lyon, France. 10.1109/IVNC49440.2020.9203448 . hal-02985178

\section{HAL Id: hal-02985178 \\ https://hal-centralesupelec.archives-ouvertes.fr/hal-02985178}

Submitted on 2 Nov 2020

HAL is a multi-disciplinary open access archive for the deposit and dissemination of scientific research documents, whether they are published or not. The documents may come from teaching and research institutions in France or abroad, or from public or private research centers.
L'archive ouverte pluridisciplinaire HAL, est destinée au dépôt et à la diffusion de documents scientifiques de niveau recherche, publiés ou non, émanant des établissements d'enseignement et de recherche français ou étrangers, des laboratoires publics ou privés. 


\section{Relative contributions to the emission within bundles of carbon nanocone emitters}

\author{
D. Mofakhami, Ph. Dessante, Ph. Teste, R. Landfried \\ Group of electrical engineering of Paris (GeePs) \\ Université Paris-Saclay, Sorbonne Université \\ CentraleSupélec, CNRS, \\ 91192, Gif-sur-Yvette, France \\ darius.mofakhami@centralesupelec.fr
}

\author{
T. Minea, B. Seznec \\ Laboratoire de Physique des Gaz et des Plasmas (LPGP) \\ Université Paris-Saclay, CNRS, \\ 91405, Orsay, France
}

\author{
J.-P. Mazellier, P.-L. Gautherin \\ Thales Research and Technology \\ 91120, Palaiseau, France
}

\begin{abstract}
Herein, an original design of electron source based on an array of carbon nanocone bundles has been modeled. The contribution to the emission within bundles is investigated using a cold emission model based on the WKB approximation. The case of a specific bundle is presented where the geometry of the nanocones leads to a change of the main emitter as the voltage is ramped up. This change is shown to alter the linearity of usual Fowler-Nordheim analysis of the simulated I(V) data.
\end{abstract}

Keywords-simulation, field emission, field emitter array, nanocones, Fowler-Nordheim plots

\section{INTRODUCTION}

$\mathrm{X}$-ray generation devices are used for a wide range of applications, from medical imaging to onboard electronics. Nevertheless, current devices generally use thermionic cathodes as electron sources, limiting their miniaturization, commutability and life-time. To overcome these limitations, the use of cold cathodes is envisioned. Achieving field emission however requires huge electric fields: above the gigavolt per meter in order to allow consequent electron tunneling. To stay at reasonable voltage levels, sharp carbon nanostructures have been considered, since their extreme aspect ratio can offer a field enhancement factor up to a few hundred at their apex [1].

In recent years, an original design of field emitter array based on carbon nanocones grown by Plasma-EnhancedChemical-Vapor-Deposition has been under development [2]. Experimental growth conditions were achieved for which bundles of a few nanocones grew on each node of the array. These bundles offer interesting results in terms of life-time and robustness but add complexity to the source. In particular, independent field emission measurement of the nanocones within a bundle is not possible. Nonetheless, idealized simulations can be used to understand the specificity of the situation. In this work, such simulations are used to get insights into the relative contribution to the emission of the emitters within a bundle.

\section{GEOMETRY AND ELECTROSTATICS}

The results shown are focused on a single bundle (Fig. 1). The geometry of the three main nanocones of the bundle is experimentally measured thanks to electron beam microscopy. In the simulation, the nanocones are defined by three parameters: the sphere radius at their apex $R_{\mathrm{s}}$, the radius at their base $R_{\mathrm{b}}$ and their height $H$. The values are displayed in Table 1 . The emitters are placed on the cathode and immersed in a macroscopic applied electric field $E_{0}$. To simulate the field enhancement around the apices of the emitters, the Laplace equation is solved. This way, we obtain the local electric field $F=\beta E_{0}$, with $\beta$ being the field enhancement factor (FEF). To evaluate the magnitude of the electrostatic screening between the emitters, we also introduce $\beta^{*}$ as the intrinsic FEF, obtained when the emitters are simulated independently. Hereafter in the document, to avoid additional notations, values of the FEF are given at the apices only. In the considered bundle, emitter 1 being the highest, it reduces the FEF of the two other emitters by a factor $\sim 2$ (Table I). Emitter 2 being much sharper, it still has a higher FEF but its sphere radius $R_{\mathrm{s}}$ is much smaller. Therefore a simulation is necessary to determine which emitter contributes the most to the current.
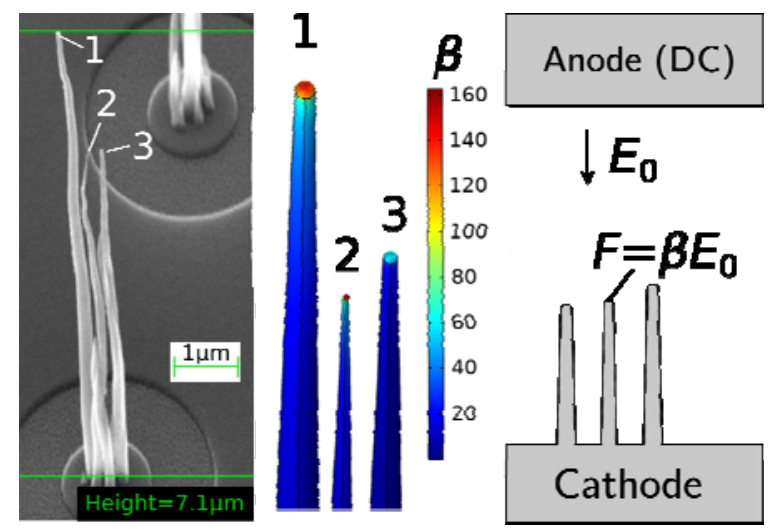

Fig. 1. Left: Electron beam microscope image of the studied bundle. Three carbon nanocones are identified and measured. Center: Colormap of the field enhancement factor $\beta$ at the emitter surface. Right: scheme of the electrostatics simulation. 
TABLE I. BUNDLE PARAMETERS

\begin{tabular}{cccccc} 
& \multicolumn{3}{c}{ Geometry } & \multicolumn{3}{c}{ Electrostatics } \\
\hline Emitter number & $\boldsymbol{H}(\boldsymbol{\mu m})$ & $\boldsymbol{R}_{\mathbf{s}}(\mathbf{n m})$ & $\boldsymbol{R}_{\mathbf{b}}(\mathbf{n m})$ & $\boldsymbol{\beta}^{*}$ & $\boldsymbol{\beta}$ \\
\hline 1 & 7.1 & 35 & 118 & 139 & 137 \\
2 & 5.5 & 9.6 & 92 & 344 & 159 \\
3 & 5.3 & 25 & 104 & 143 & 63 \\
\hline
\end{tabular}

\section{EMISSION AND FOWLER-NORDHEIM PLOTS}

The calculation of the emitted current density is done using a WKB type approximation and a numerical estimation of the elliptic functions. The current emitted by each nanocone is then calculated by integrating the current density over their surface. Fig. 2 shows the respective contributions of each emitter relatively to the total current as a function of the applied electric field. At electric field below $25 \mathrm{~V} / \mu \mathrm{m}$, emitter 2 dominates the emission, as its FEF $\beta_{2}=159$ is higher than the FEF of emitter $1, \beta_{1}=137$. When the field is ramped up, however, the emission surface of emitter 2 is limited by its smaller sphere radius $R_{\mathrm{S}}=9.6 \mathrm{~nm}$, while the contribution to the emission of emitter 1 increases faster (Fig. 2). Above $35 \mathrm{~V} / \mu \mathrm{m}$, emitter 1 compensates its lower current density by a larger emission surface, resulting in an overall higher contribution to the total current of the bundle (Fig. 3).

This change of the prevalent emitter over the applied electric field has a consequence on the linearity of the I(V) characteristic in the Fowler-Nordheim coordinates. If we try to

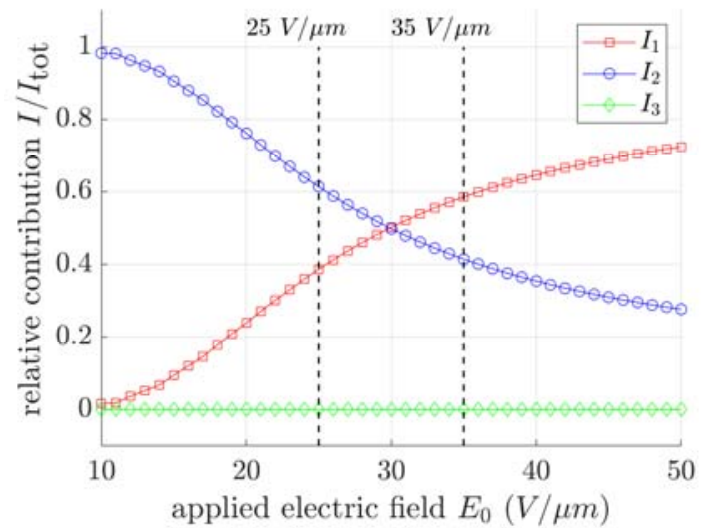

Fig. 2. Variation over the applied electric field of the emitter relative contribution to the total current of the bundle $I_{\text {tot }}$

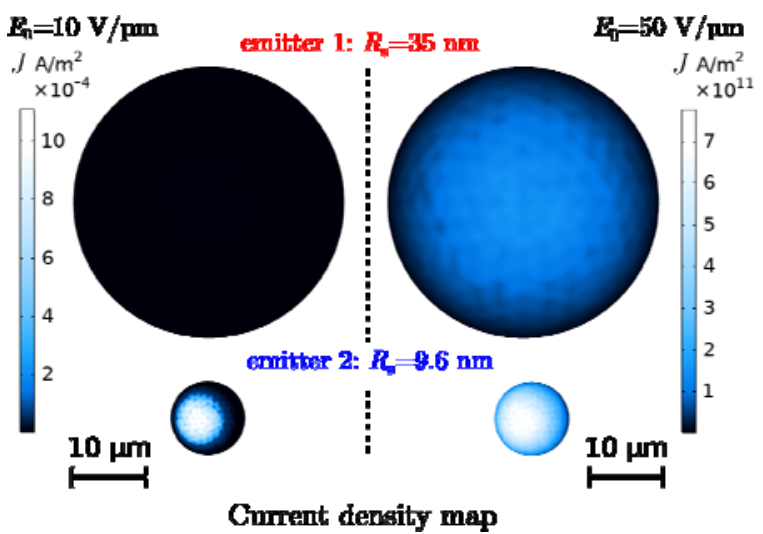

Fig. 3. Current density map for emitter 1 and 2. The central dash line separates the color map for $E_{0}=10 \mathrm{~V} / \mu \mathrm{m}$ (left) and the color map for $E_{0}=50 \mathrm{~V} / \mu \mathrm{m}$ (right)

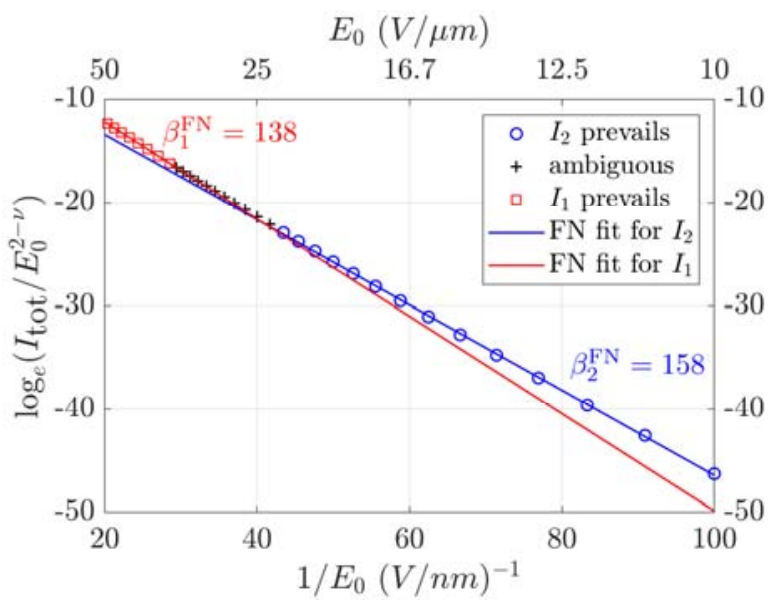

Fig. 4. I(V) characteristic for the simulated bundle in the Fowler-Nordheim coordinates, as prescribed by $\mathrm{K}$. Jensen [5]. The $v$ exponent is related to the

extract a FEF of our bundle over the range $10-50 \mathrm{~V} / \mu \mathrm{m}$, we will in fact mix the FEF of the two contributing emitter 1 and 2. Using the Fowler-Nordheim coordinates prescribed by $\mathrm{K}$. Jensen [3], we get $\beta^{\mathrm{FN}}=151$ (plot not shown), an intermediary value between $\beta_{1}=137$ and $\beta_{2}=159$. When we however use separated extrapolations at high and low electric field we observe more linear fits and are able to retrieve close values for the FEF of emitter $1\left(\beta_{1}=137\right)$ and $2\left(\beta_{2}=159\right)$ : in red, between 35 and $50 \mathrm{~V} / \mu \mathrm{m}$, we extract $\beta^{\mathrm{FN}}{ }_{1}=138$ and in blue between 10 and $25 \mathrm{~V} / \mu \mathrm{m}$ we find $\beta^{\mathrm{FN}}{ }_{2}=158$ (Fig. 4). This specific example gives insights into how the geometry variations related to the chaotic growth of the emitters can impact the theoretical extrapolations from experimental data.

\section{CONCLUSION}

We have highlighted through modeling results how a change in the prevalent emitter within the bundle can affect the linearity of the I(V) characteristic in the Fowler-Nordheim coordinates. Besides, this change of the prevalent emitter has been related to a specific geometry observed in experiment. Due to the chaotic growth of the emitters during the fabrication of the source, we do think this specific situation can be a frequent case.

To go further, we plan to build empirical distributions of the nanocone geometry then simulating the emission of many bundles sampled from these distributions. We will then investigate the implication of the growth statistics on experimental-like measurement.

\section{REFERENCES}

[1] Y. Saito, Carbon Nanotube and Related Field Emitters: Fundamentals and Applications. John Wiley \& Sons, 2010.

[2] L. Sabaut, P. Ponard, J.-P. Mazellier, et P. Legagneux, « Unprecedented current modulation with in-plane gated field emitters », in 2016 29th International Vacuum Nanoelectronics Conference (IVNC), Vancouver, BC, Canada, juill. 2016, p. 1国2, doi: 10.1109/IVNC.2016.7551457.

[3] K. Jensen, Introduction to the Physics of Electron Emission. Chichester, UK: John Wiley \& Sons, Ltd, 2017. 